\title{
Successful treatment of Subclavian artery thrombosis with gangrene of fingers- a case report
}

\author{
Md. Rezwanul Hoque ${ }^{1}$, Sabrina Husain ${ }^{1}$, \\ Md. Alauddin 1 , Zerzina Rahman ${ }^{2}$ \\ ${ }^{1}$ Department of Cardiac surgery, Bangabandhu Sheikh Mujib Medical University, Dhaka \\ ${ }^{2}$ Department of Cardiac Anaesthesiology, Bangabandhu Sheikh Mujib Medical University, Dhaka \\ Address of correspondence \\ Dr. Md. Rezwanul Hoque Bulbul, Associate Professor, Cardiac surgery, Bangabandhu Sheikh Mujib Medical University, Dhaka \\ E-mail: drrhbulbul@yahoo.com
}

\begin{abstract}
Mr. X, a smoker, normotensive, nondiabetic male patient of 24 years of age, presented with acute severe pain of the left upper extremity for 2 days. He had discoloration of the thumb and index finger for which he consulted a quack doctor who made an incision over the blackened area to drain subcutaneous collection and gave antibiotic and analgesics. There was no bleeding from the site of incision and regular dressing could not improve his condition. After waiting for 15 days without any improvement, he reported to a private clinic in Dhaka. Thorough physical examination and investigation revealed subclavian artery thrombosis due to cervical rib. The thoracic outlet syndrome was relieved by sclaneous muscle division and excision of the cervical rib, subclavian artery thrombectomy was possible through transbrachial route even after more than 2 weeks.
\end{abstract}

\section{Introduction}

Subclavian artery thrombosis may be due to external compression from skeletal or muscular components at thoracic outlet or due to intimal injury from atherosclerotic process or sometimes a hypercoagulable state may be responsible. Embolism from a proximal lesion present with acute ischaemia but chronic lesion may be asymptomatic due to well developed collateral circulation. ${ }^{1}$ Overuse of the arm particularly unaccustomed exercise may predispose to subclavian artery thrombosis. In case of thoracic outlet syndrome sometimes neural or venous component signs predominate. Acute ischemia of the upper limb is easy to diagnose but in case of chronic ischaemia the symptoms may be vague, sometimes symptoms of subclavian steal syndrome predominate giving rise to diagnostic confusion. A thorough clinical evaluation, signs and symptoms of acute or chronic ischaemia, thoracic outlet compression, laboratory study to exclude hyper coagulable state, radiological study of the thoracic outlet to exclude cervical rib, MRI scan of the thoracic outlet, MR angiography of the subclavian vessel, nerve conduction study, subclavian arteriography establishes the diagnosis. In acute ischemia catheter based therapy with thrombolytic, Fogarty embolectomy followed by anticoagulant therapy is the mainstay of treatment. In chronic ischaemia if the patient is asymptomatic due to good collateral flow surgical treatment is usually not necessary, but in symptomatic cases cervical or first rib excision, sclaneus muscle division, bypass grafting (Carotid-subclavian, subclavian to subclavian, axillary to axillary) apart from anticoagulant therapy may be needed.

\section{Case report}

Mr. X, a smoker, normotensive, nondiabetic male patient of 24 years of age, presented with acute severe pain of the left upper extremity for 2 days. He had discoloration of the thumb and index finger for which he consulted a quack doctor who made an incision over the blackened area to drain subcutaneous collection and gave antibiotic and analgesics. There was no bleeding from the site of incision and regular dressing could not improve his condition. After waiting for 15 days without any improvement, he reported to a private clinic in Dhaka. A thorough physical examination revealed gangrene of the left thumb and index finger, absence of pulse in the axillary, brachial, radial and ulnar arteries, intact motor and sensory functions in all the three digits except the thumb and index fingers. Haematological investigations revealed no coagulopathy, biochemical examinations were normal. Radiological examination revealed cervical rib on the affected side. Duplex scan confirmed absence of pulses from subclavian to distal arteries but venous outflow was normal. Arteriogram showed distal subclavian and proximal axillary artery occlusion with nonvisualisation of distal arteries. 
Based on these finding a decision for surgical intervention was taken and on $2^{\text {nd }}$ April, 2008 operation was done. The supraclavicular approach was used, the sclaneous anterior muscle was divided, cervical rib was divided in the middle and was removed using bone nibbler, the subclavian artery was freed from any compressive effect, the site of occlusion was found difficult to manage through this approach. So the Brachial artery was exposed at the elbow, transverse arteriotomy was done and by Fogarty catheter a four inch organized thrombus was removed. Proximal flow was established, distal embolus was excluded by Fogarty embolectomy catheter, the arteriotomy was closed with $6{ }^{\prime} 0$ ' prolene suture after proper deairing. Conservative amputation of the thumb and index finger was done. The patient recovered well but needed secondary skin grafting on the amputation wound. Patient was put on oral warfarin therapy for subsequent 6 months. Follow up at 6 months revealed presence of pulses in all distal vessels with good functional status in all the three fingers.

\section{Discussion}

Acute arm ischaemia accounts for one-fifth of all episodes of acute limb ischaemia. It occurs twice as often in females as in males. Brachial embolectomy is the most common treatment. After successful brachial embolectomy, $95 \%$ of patients are symptom free ${ }^{2}$, however, the operative mortality may be as high as $12 \% .^{3}$ although conservative management are sometimes successful in patients with acute limb ischaemia, every effort should be made to restore blood flow in patients having reasonable life expectancy. ${ }^{4}$

Surgery to correct subclavian artery thrombosis is the treatment of choice. Some authors have achieved good result by using thrombolytic to treat acute occlusion of the axillary artery or brachial artery but in a recent study the success rate of this procedure is only 55\%. ${ }^{5,6}$ Fogarty embolectomy catheter may be used for acute thrombosis. In this case it was successful even after more than 2 weeks. Interventions include catheter-based procedures and formal operative procedures. Embolisation recurs after successful emboletomy in about one-third of the patients if systemic anticoagulation is not used. It may even recur in about $11 \%$ of cases after oral anticoagulation. ${ }^{7}$

Occasionally, the hand continues to appear ischaemic after successful embolectomy. This persistent ischaemia is caused either by an unrecognized inflow lesion or by embolization to the digital arteries that has been occurring over an extended period. In these patients, an arch aortogram with selective views of the affected arm should be performed immediately after operation. Any lesion in the subclavian artery or innominate artery can be treated by angioplasty or stenting. ${ }^{8}$

Most patients with chronic subclavian artery stenosis or occlusion require no treatment. In many cases overt symptoms are absent and the diagnosis is made serendipitously when a reduced pulse pressure is encountered in one arm. For patients with symptomatic lesion, balloon angioplasty with stent insertion is currently the treatment of choice. Angioplasty and stenting of stenotic and even occluded arteries have been undertaken successfully with adequate patency rates and minimal morbidity. These interventions are particularly appropriate for atherosclerotic arteries. 9,10

Subclavian artery occlusion secondary to thoracic outlet syndrome or muscular compression is treated by excision of the anatomical structure compressing the artery, whether muscle or bone. The artery may or may not require additional reconstruction, depending on the presence or absence of intimal damage. ${ }^{1}$

The occluded artery may require a bypass procedure, depending on the location of the occlusion or the presence of a subclavian steal syndrome. The bypass options include subclavian-carotid, subclavian-subclavian, and axillaryaxillary bypasses. Another possible bypass option is transposition of the subclavian artery to the ipsilateral carotid artery.

In any operative procedure for the problem, care must be taken to protect the thoracic duct from damage.

The results from stenting procedures on the subclavian artery document an $87 \%$ patency rate after 3 years. Operative outcomes demonstrate about a $90 \%$ patency rate after 5 years. ${ }^{1}$

\section{References}

1. Mary C Mancini. Subclavian Artery Thrombosis. eMedicine $\underline{\text { Specialties }}>$ Thoracic Surgery $>$ Vascular, Last Updated: Jan 15, 2008, http://journal.medscape.com/mjm

2. Hernandez-Richter T, Angele MK, Helmberger T et al: Acute ischaemia of the upper extremity: long term result following thromboembolectomy with Fogarty catheter. Langenbecks Arch Surg 386: 261, 2001.

3. Wirsing P, Andriopoulos A, Botticher R. arterial embolectomies in the upper extremity after acute occlusion: report on 79 cases. J Cardiovasc Surg(Torino) 24;40, 1983.

4. Galbraith K, Collin j, Morris PJ, et al. Recent experience with arterial embolism of the limb in a vascular unit. Ann R Coll Surg Engl 67:30, 1985. 
5. Widlus DM, Venbrux AC, Benenati JF, et al. Fibrinolytic therapy for upper extremity arterial occlusions. Radiology 175:393, 1990.

6. Cejna M, Salomonowitz E, Wohlschlager H, et al. rt-PA thrombolysis in acute thromboembolic upper-extremity arterial occlusion. Cardiovasc intervent Radiol 24: 218, 2001.

7. Stonebridge PA, Clason AE, Duncan AJ, et al. Acute ischaemia of the upper limb compared with acute lower limb ischaemia, a 5 year review. B J Surg 76: 515, 1989.

8. Byrne J, Paty Philip SK, Darling RC. Upper extremity revascularization procedures, ACS surgery- principles and practice, vascular system, 6: 1-11, 2006.

9. Woo EY, Fairman RM, Velaquez OC, et al. Endovascular therapy of symptomatic innominate-subclavian arterial occlusive lesion.Vasc Endovascular Surg 40:27,2006.

10. Brounzots EN, Petersen B,Binkert C, et al.Primary stenting of subclavianand innominate artery occlusive disese: a single centre's experience.Cardiovasc Intervent radiol 27:616, 2004.
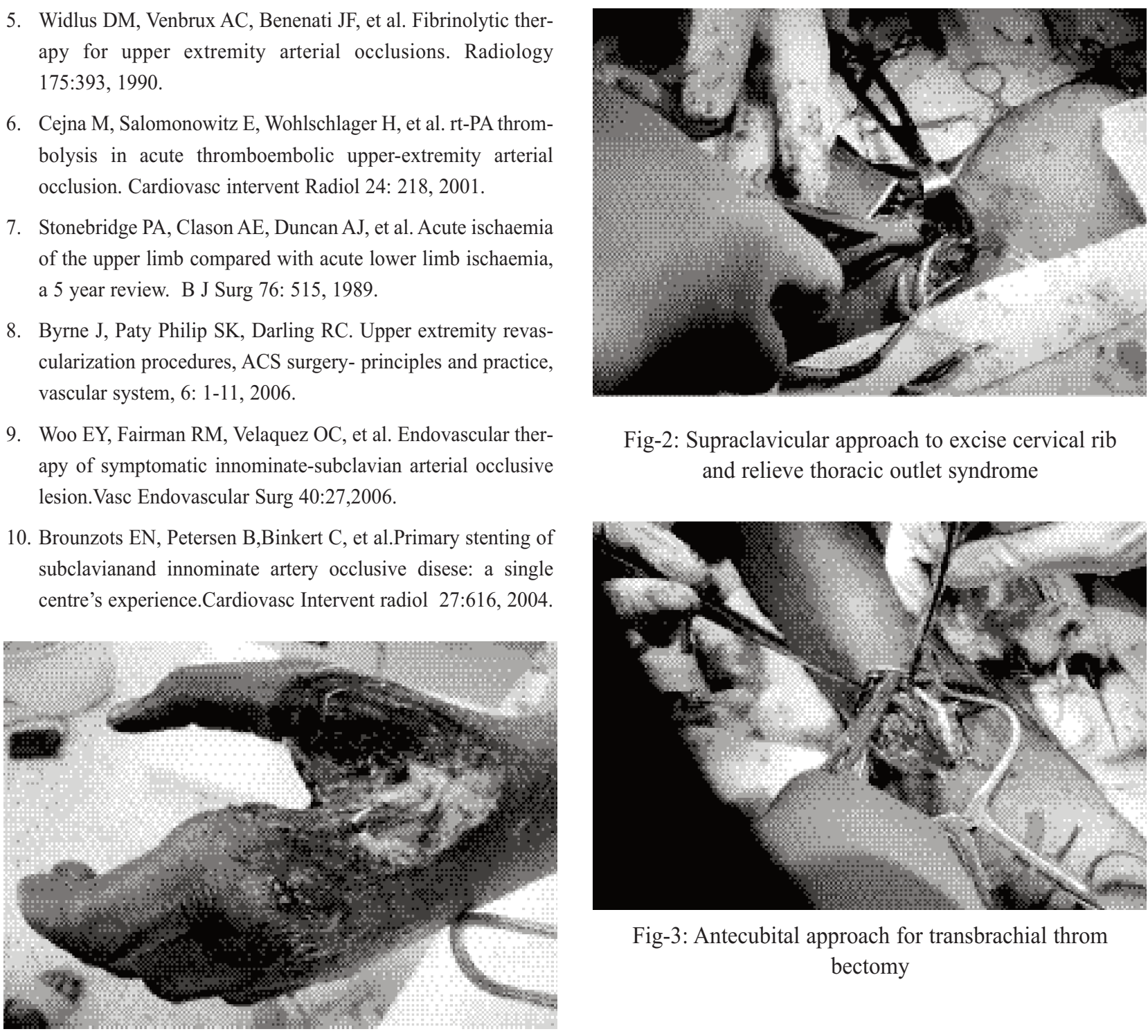

Fig-2: Supraclavicular approach to excise cervical rib and relieve thoracic outlet syndrome

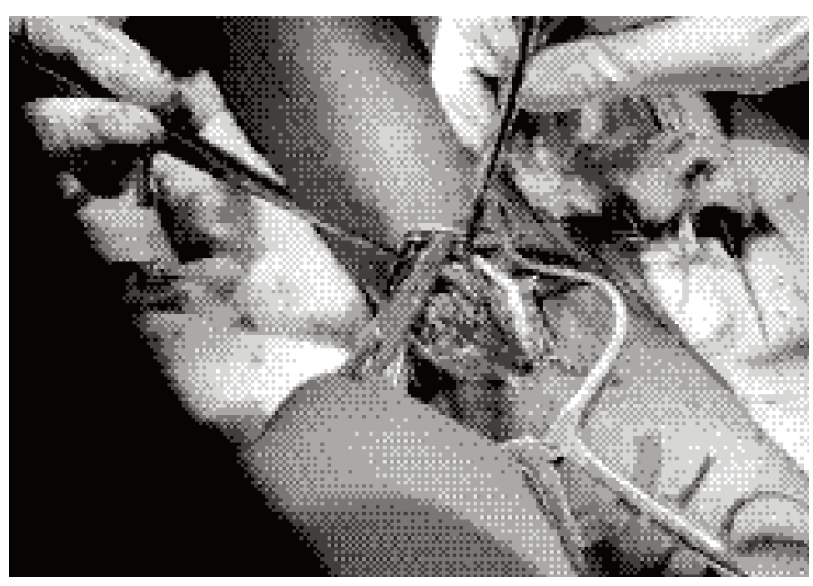

Fig-3: Antecubital approach for transbrachial throm bectomy

Fig-1 : Ischaemic gangrene resulting from subclavian artery thrombosis 